\title{
Primary orbital Ewing's sarcoma: report of a case and review of the literature
}

\section{D.S.C. Lam \\ L.L. Cheng \\ J.S.K. Ng \\ W.W.L. Poon \\ Department of \\ Ophthalmology and Visual \\ Sciences \\ C.K. Li \\ K.W. Chik \\ P.M.P. Yuen \\ Department of Pediatric \\ Oncology}

J.G.C. Teo

Department of Anatomical

\& Cellular Pathology

W.H. Kwan

Department of Clinical

Oncology

The Chinese University of

Hong Kong

Prince of Wales Hospital

Shatin, N.T., Hong Kong

Dr Dennis S.C. Lam, FRCS, FRCOphth

Department of

Ophthalmology and Visual

Sciences

The Chinese University of Hong Kong

Prince of Wales Hospital

Shatin, N.T., Hong Kong

Tel: (+852) 26322881

Fax: (+852) 26482943

e-mail:

dennislam@cuhk.edu.hk

Presented in part at the

Hong Kong

Ophthalmological

Symposium, December

1997, Hong Kong

Supported in part by the Mrs Annie Wong Eye

Foundation

Received: 23 March 1998 Accepted in revised form: 23 October 1998

\begin{abstract}
Primary orbital Ewing's sarcoma is a very rare condition. Since the first case was reported in 1950 , only 7 other cases have been reported in the English literature. Herein we describe the ninth, the only bilateral and the youngest case of primary orbital Ewing's sarcoma occurring in a 2-year-old boy, who presented to us with bilateral painless proptosis. Tissue biopsy of the tumour was obtained through the nasal sinus. Immunohistological studies of the biopsy tissue confirmed the diagnosis of Ewing's sarcoma. No distant site of the tumour was found so this was considered a primary orbital tumour. Combined chemotherapy and radiotherapy without surgical resection achieved an encouraging result in that the patient has remained in remission for $\mathbf{3 0}$ months after completion of treatment. The successful use of combined chemotherapy and radiotherapy, without surgery, adds further support to evidence that surgical excision may be avoided in selected cases of primary orbital Ewing's sarcoma.
\end{abstract}

Key words Ewing's sarcoma, Multi-drug chemotherapy, Orbital, Primary, Proptosis, Radiotherapy

Ewing's sarcoma is thought to be the least differentiated of a group of small cell neoplasms with various degrees of neuroectodermal differentiation. ${ }^{1}$ It most commonly arises in the diaphysis of a long bone, or in the ribs, pelvis or vertebrae. ${ }^{2}$ Primary orbital involvement or localisation is very rare. Since 1950, when Harbert and Tabor ${ }^{3}$ reported the first case of primary orbital Ewing's sarcoma, only 7 others have been reported (Table 1).

A definitive cure of Ewing's sarcoma can be achieved only if all local and systemic cancer cells are eradicated. ${ }^{2}$ Tremendous progress in the treatment of Ewing's sarcoma has been made in the past 25 years. The development of multi-agent chemotherapy protocols has increased the 5 year survival rate of Ewing's sarcoma patients from $5-10 \%$ twenty years ago, to more than $70 \%$ now. ${ }^{4}$
DENNIS S.C. LAM, CHI K. LI, LULU L. CHENG, JENNIFER G.C.TEO, KI W. CHIK, WING H. KWAN, JOAN S.K. NG, WILLIAM W.L. POON, PATRICK M.P. YUEN

Surgical resection of the primary tumour is still considered the treatment of choice for local control, but this often results in cosmetic mutilation and physical handicap. The efficacy of radiotherapy for local control is still under debate, even though these tumours are usually radiosensitive. For systemic control of nonpelvic Ewing's sarcoma, ${ }^{5}$ a high-dose multidrug chemotherapy regimen (adriamycin, cyclophosphamide, vincristine and actinomycin) has been shown to be effective in achieving a high rate of recurrence-free survival.

In our case we used a combination of multidrug chemotherapy and local radiotherapy, in accordance with the protocol of the 1992 European Intergroup Co-operative Ewing's Sarcoma (EICES) study. ${ }^{6}$ An encouraging result was achieved in that the patient has remained well, with no recurrence, for 30 months after completion of treatment.

\section{Case report}

A 2-year-old boy with a 1 month history of bilateral painless proptosis was referred to our institution for further investigation and management. The child had no prior medical problems and, more specifically, no history of thyroid or renal disease. Family history was non-contributory and the boy was an only child.

Examination revealed bilateral proptosis.

Visual acuity was hand movement and 20/50 in the right and left eyes, respectively. There was an afferent pupillary defect of the right eye. Fundus examination revealed no papilloedema. Both optic discs were pink and appeared normal. The range of extraocular movement was full, the cranial nerves were intact and no other neurological deficits were found.

The patient was admitted for further investigations. A complete blood count showed a normal level of haemoglobin $(13.5 \mathrm{~g} / \mathrm{dl})$, white cell count $\left(9 \times 10^{9} / 1\right)$ and platelet count $\left(342 \times 10^{9} / 1\right)$. Erythrocyte sedimentation rate was $36 \mathrm{~mm} / \mathrm{h}$. Liver function tests, electrolytes, urea and creatinine levels were all within normal limits. A computed tomography (CT) scan showed an enhancing soft tissue mass 


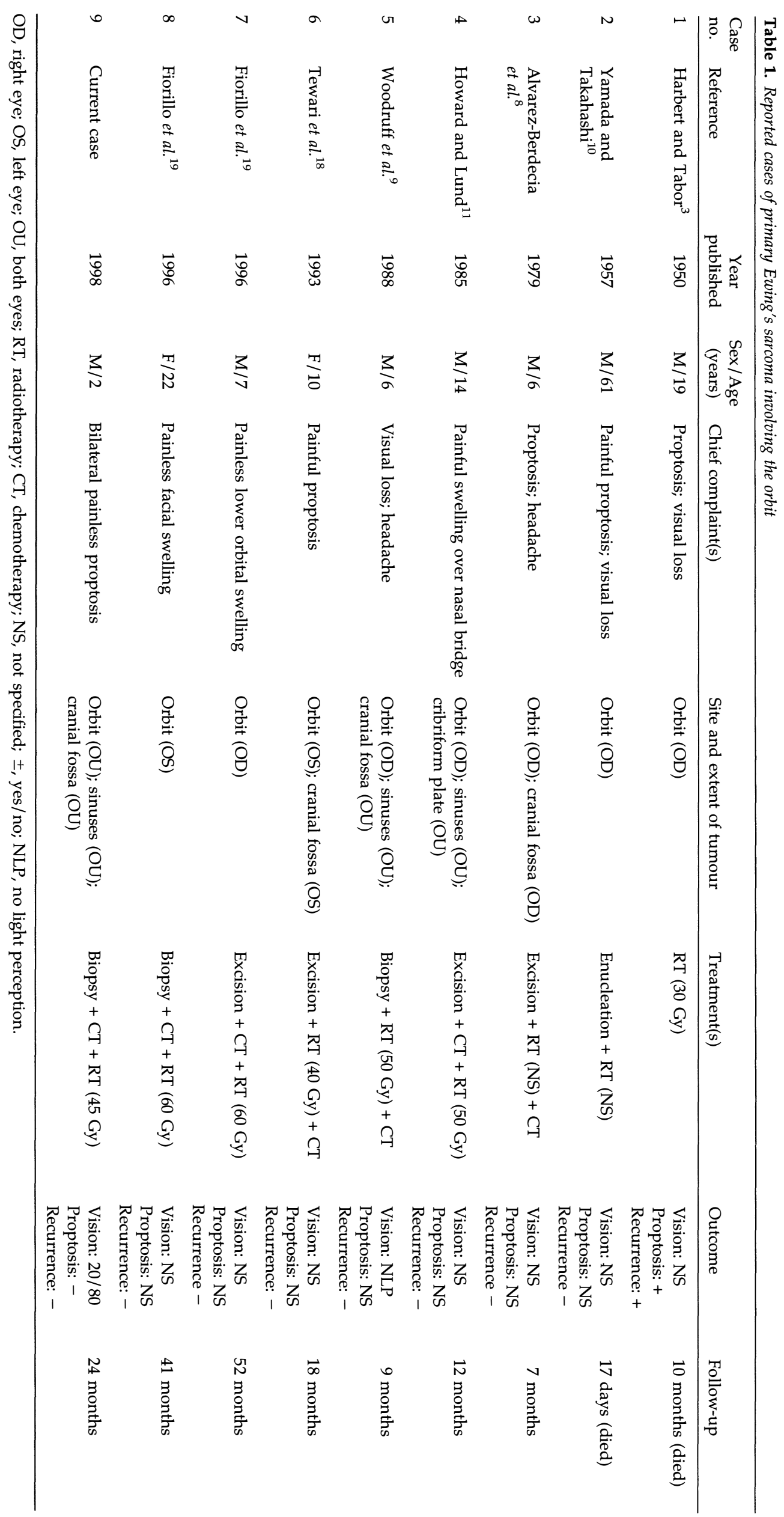




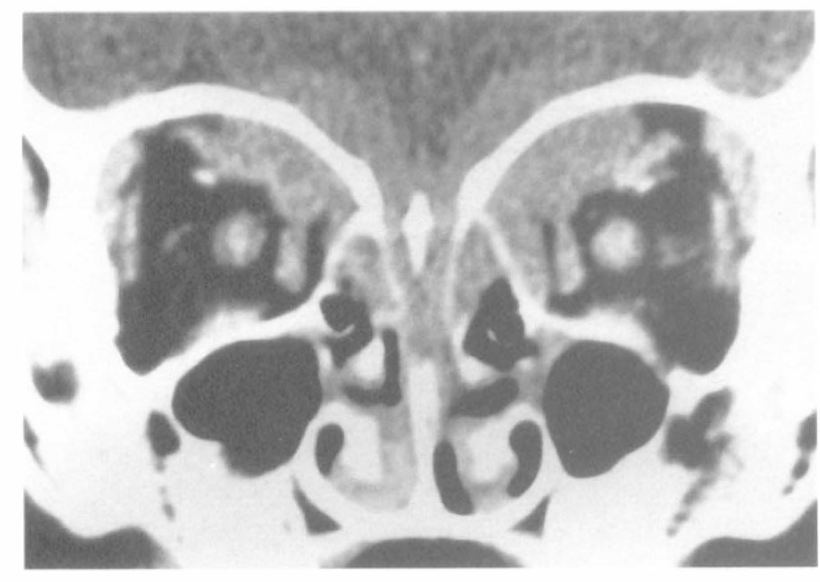

Fig. 1. Pre-treatment CT scan showing the extensive tumour mass involving both orbits, ethmoidal sinuses and the anterior cranial fossa.

involving the medial orbits, the ethmoidal sinuses and the anterior cranial fossa (Fig. 1). Tissue biopsy of the tumour was obtained through the nasal sinus, and standard as well as special immunohistochemical stains were used to identify the nature of the tumour. Histological examination revealed the mass to be a small round cell tumour. Haematoxylin and eosin stain showed the presence of sheets of monotonous small round cells that infiltrated the lamina propria (Fig. 2). These cells were positive for the periodic acid-Schiff (PAS) stain and the PAS staining was diastase sensitive. Immunohistochemical stains showed focal positivity for neurone-specific enolase and diffuse positivity for the MIC2 marker (Fig. 3). The MIC2 marker is used to detect MIC2 protein, whose production is controlled by the MIC2 gene located at the short art of the $X$ and $Y$ chromosomes; it may present on the surface of tumour cells of both Ewing's sarcoma and peripheral neuroepitheliomas and has been shown to be present in the vast majority Ewing's sarcomas. ${ }^{7}$

Once the diagnosis of Ewing's sarcoma was made, staging investigations were initiated. Ultrasonography of the abdomen showed no liver or lymph node involvement, but a bone scan did show increased uptake of radioisotope in the orbital and ethmoidal areas. CT of

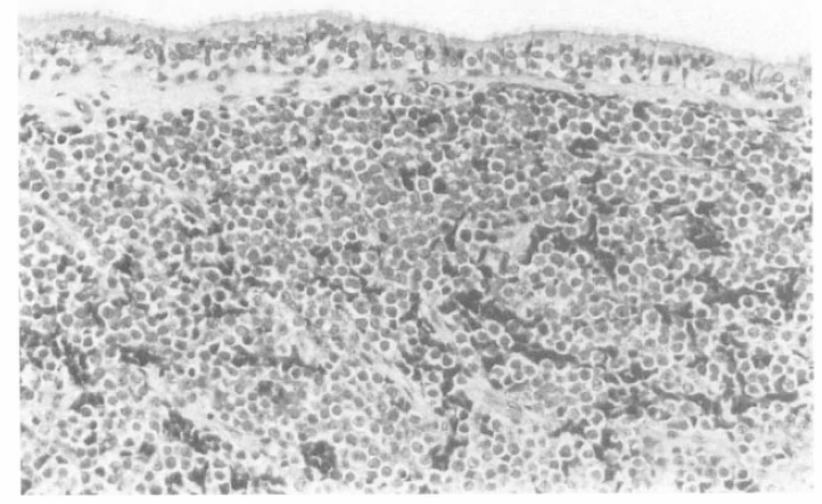

Fig. 2. Histology of the Ewing's sarcoma showing sheets of monotonous small round cells with infiltration of the lamina propria (haematoxylin $\mathcal{E}$ eosin; original magnification, $\times 150$ ).

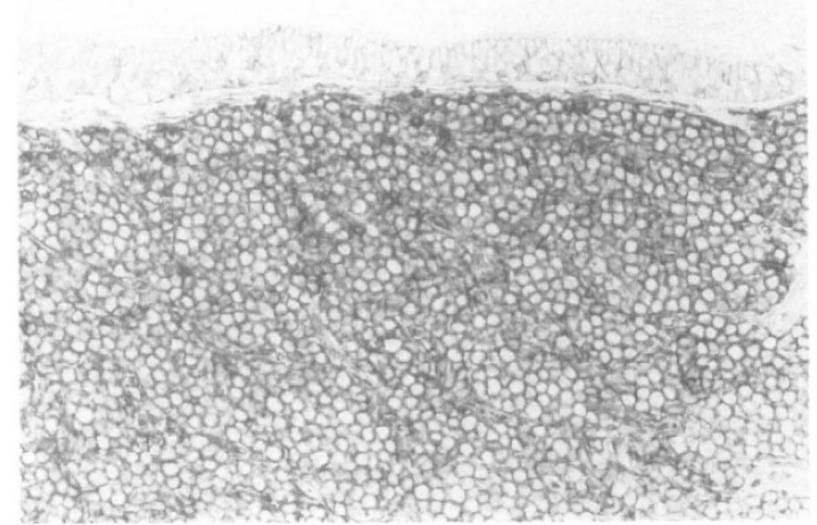

Fig. 3. The tumour cells show strong diffuse surface immunoreactivity to the MIC2 marker (stain, avidin-biotin-peroxidase; original magnification, $\times 150)$.

the thorax and abdomen showed no lung or lymph node involvement, and bone marrow biopsy was also negative.

According to the 1992 EICES study protocol, the treatment of Ewing's sarcoma is divided into three phases. Phase one is induction chemotherapy composed of four courses of chemotherapy, each lasting for 3 weeks. Phase two consists of definitive local treatment, which can be surgical excision, radiotherapy or both. Phase three is ten courses of chemotherapy with each course lasting 3 weeks. Completion of the treatment thus takes 42 weeks.

Patients with a confirmed tissue diagnosis are first classified into standard or high risk. High risk refers to patients with metastasis or a tumour volume greater than $100 \mathrm{ml}$. According to the 1992 EICES study protocol, patients in each group are randomised to one of two arms. Standard-risk patient will first receive VAIA (vincristine $1.5 \mathrm{mg} / \mathrm{m}^{2}$, adriamycin $20 \mathrm{mg} / \mathrm{m}^{2}$, ifosfomide $2000 \mathrm{mg} / \mathrm{m}^{2}$ and actinomycin $0.5 \mathrm{mg} / \mathrm{m}^{2}$ ) as induction chemotherapy and will then be randomised to receive either VACA (vincristine $1.5 \mathrm{mg} / \mathrm{m}^{2}$, adriamycin $20 \mathrm{mg} / \mathrm{m}^{2}$, cyclophosphamide $1200 \mathrm{mg} / \mathrm{m}^{2}$ and actinomycin $0.5 \mathrm{mg} / \mathrm{m}^{2}$ ) or to continue the VAIA chemotherapy regimen. High-risk patients are randomised to receive either VAIA or EVAIA (etoposide $150 \mathrm{mg} / \mathrm{m}^{2}$, vincristine $1.5 \mathrm{mg} / \mathrm{m}^{2}$, adriamycin $20 \mathrm{mg} /$ $\mathrm{m}^{2}$, ifosfomide $2000 \mathrm{mg} / \mathrm{m}^{2}$ and actinomycin $0.5 \mathrm{mg} / \mathrm{m}^{2}$ ) for the entire 14 courses of chemotherapy.

Our patient was classified in the high-risk group because of the large volume of the tumour. One week after the tissue diagnosis was made, he was randomised to receive the VAIA regimen. In view of the very extensive involvement of the tumour and the anticipated mutilating effect of surgical excision, radiotherapy rather than surgery was contemplated for local tumour control. Radiotherapy was set to be started after the induction chemotherapy, but was delayed for a long time as his parents were afraid of its potential side effects.

Radiotherapy was ultimately started after completion of the entire 42 weeks of chemotherapy. 


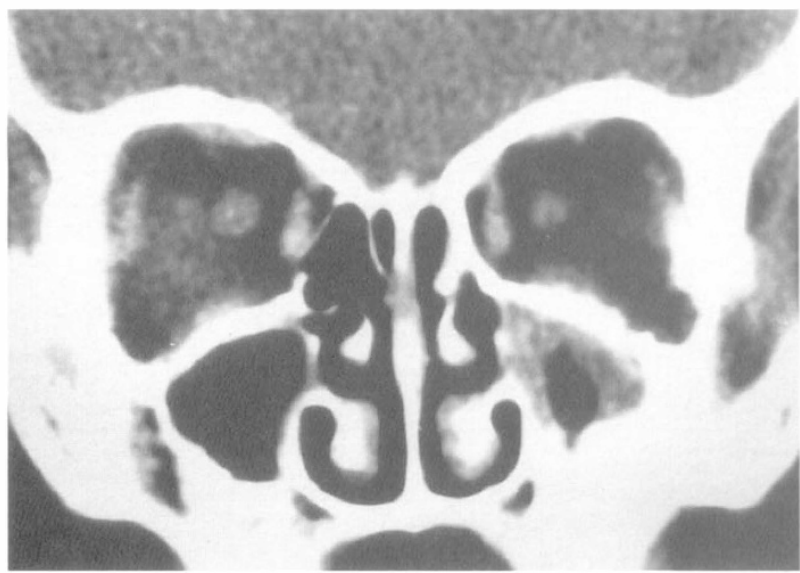

Fig. 4. Post-treatment CT scan showing absence of the tumour mass.

Because we felt that the patient's parents might refuse radiotherapy, thus leaving chemotherapy as the only treatment, we added etoposide to the four drugs (i.e. therapy was changed from the VAIA to the EVAIA regimen) after the first ten courses.

When finally agreed to by the parents, the radiotherapy was delivered through a pair of left and right opposing portals; the radiation field was $5 \times 7 \mathrm{~cm}$, and included the initial tumour volume with its superior border placed at about $1.2 \mathrm{~cm}$ above the roof of the sphenoid sinus. Divergent blocks protected regions outside the treatment field. The total dose of radiation was $45 \mathrm{~Gy}$, delivered in fractions of $1.8 \mathrm{~Gy}$ per day, 5 days a week. Radiation was completed in 5 weeks.

Subsequent follow-up CT scans (Fig. 4) showed no evidence of residual tumour and only minimal reactive inflammatory fibrosis of the orbits. The patient's visual acuity improved to 20/80 and 20/40 in the right and left eyes respectively. At the time of writing he has been in remission for 30 months since the last dose of radiotherapy, and apart from very slight temporal disc pallor in both eyes, no radiation-induced cataract, keratitis, retinopathy, neuropathy, abnormal orbital growth or secondary malignant tumour has been observed.

\section{Discussion}

Primary Ewing's sarcoma rarely involves the orbit and, in fact, less than $5 \%$ of the cases of Ewing's sarcoma arise anywhere in the skull region. ${ }^{3}$ Since 1950 , when the first case of Ewing's sarcoma of the orbit was described, only 7 other cases have been reported in the literature (Table 1). Among reported cases there was male-to-female ratio of 6 to 2, with a tendency for involvement of the right orbit (right: left, 6:2). The mean age at presentation was 18 years (range 6-61 years), with most patients being diagnosed in late childhood or early adulthood. The two youngest cases reported previously, by Alvarez-Berdecia et al. $^{8}$ and Woodruff et al., ${ }^{9}$ were both 6 years old. The oldest patient was a 61-year-old man reported by Yamada and Takahashi. ${ }^{10}$ Our case is the youngest of all the previously reported cases of primary orbital Ewing's sarcoma, being only 2 years old at the time of diagnosis. Moreover, of the 8 previously reported cases, no bilateral orbital involvement was reported, so this is the first case with bilateral orbital involvement. Furthermore, this is one of the cases with the most extensive involvement (both orbits, both ethmoidal sinuses and anterior cranial fossa), there being only 2 other cases with orbital, sinus and cranial fossa involvement. ${ }^{9,11}$

The most common presentation of primary orbital Ewing's sarcoma is proptosis, with or without pain, but other presenting symptoms include visual loss and headache. Major differential diagnoses for bilateral proptosis in a 2-year-old child include orbital trauma, infection, inflammation, dysthyroid eye disease, cavernous sinus disorder, and primary or secondary tumours. Primary tumours, such as rhabdomyosarcoma, optic glioma, lymphangioma and infiltrative lymphoma, although uncommon, have to be considered. Secondary tumours, such as mesenchymal cell chondrosarcoma, neuroblastoma and histiocytosis $X$, are also rare but possible causes.

Ewing's sarcoma is the least differentiated of a group of small round cell tumours that have various degrees of neuroectodermal differentiation. Other members of this group are peripheral neuroepitheliomas and neuroblastomas. ${ }^{12}$ Difficulty had been encountered in distinguishing Ewing's sarcoma from other small round cell tumours when they occur in the orbital region., ${ }^{5,14}$ Exact diagnostic criteria for distinguishing Ewing's sarcoma from peripheral neuroepithelioma have not been generally agreed upon. Histologically, both tumours consist of small round cells with scanty cytoplasm and fine chromatin; cytogenetically, both possess translocation abnormality $\mathrm{t}(11,22)$. For rather general classification, tumours with Homer-Wright rosettes and definite positivity for more specific neural markers such as synoptophysin or neurofilament, or ultrastructural demonstration of dense core granules, are classified as peripheral neuroepitheliomas. Otherwise, both Ewing's sarcoma and malignant peripheral neurectodermal tumours react positively to the MIC2 marker and to neurone-specific enolose. ${ }^{14}$

In the past 25 years marked progress has been made in the treatment of Ewing's sarcoma. The mainstay of treatment for Ewing's sarcoma is surgery for local removal of the tumour and chemotherapy for control of distant spread. ${ }^{15,16}$ Chemotherapy has been very effective in eradicating subclinical distant metastastis, which would otherwise develop in an estimated 75-85\% of cases within the first 2 years. ${ }^{2}$ This has increased the 5 year survival rate tremendously, from $5 \%$ in the past to more than $70 \%$ now. ${ }^{2,4,5}$

For local tumour control, surgery may not be the treatment of choice if its mutilating effect is expected to be substantial. Radiotherapy is an alternative, but may not be as effective as surgery. For systemic control, a clinical trial of multi-drug chemotherapy has shown this treatment to be useful in improving the relapse-free survival to $73 \%$ at a median follow-up of 5.6 years. ${ }^{17}$ The chemotherapy regimen first studied consisted of high- 
dose VAIA, begun 2-4 weeks after histological diagnosis. In 1990, a group of German investigators suggested intensive chemotherapy with VAIA followed by either surgery, radiotherapy or both. ${ }^{16}$ This protocol was further consolidated into the new EICES (1992) study protocol. $^{6}$

Among the 8 previously reported cases, surgical excision was performed in $5.8,10,11,18,19$ Surgical biopsy was performed on 2 of the patients, who were later treated successfully by combined radiotherapy and chemotherapy without further surgery. ${ }^{9,19}$ In our patient, because the tumour involvement was very extensive, surgical debulking would have resulted in much mutilation and de-functioning, so high-dose radiation therapy was used for local control. Radiotherapy, however, is not without risk. Numerous complications, including radiation cataract, retinopathy, abnormal orbital growth and secondary tumours, can occur. ${ }^{20,21}$ Although no such complications have been noted in our patient at the time of writing, close observation is essential. Overall, the results have been very encouraging in that this child has remained in remission for 30 months after completion of treatment, and has enjoyed good functional and cosmetic outcome.

The authors thank Dr Michael C.F. Tse, FRCS, for referring this patient to us.

\section{References}

1. Jaffe R, Santamaria M, Yunis EJ, et al. The neuroectodermal tumour of bone. Am J Surg Pathol 1984;8:885-98.

2. Jereb B, Ong RL. Redefined role of radiation in combined treatment of Ewing's sarcoma. Paediatr Haematol Oncol 1986;3:111-8.

3. Harbert F, Tabor GL Jr. Ewing's tumour of the orbit: report of two cases. Am J Ophthalmol 1950;33:1219-25.

4. Vlasak R, Sim FH. Ewing's sarcoma. Orthop Clin North Am 1996;27:591-603.

5. Wilson WB, Roloff J, Wilson HL. Primary peripheral neuroepithelioma of the orbit with intracranial extension. Cancer 1988;62:2595-601.

6. Craft AW, Jurgens H. European Intergroup Co-operative Ewing's Sarcoma Study 1992. EICESS 92 Protocol 1992;17-49.
7. Lee CS, Southey MC, Waters K, et al. EWS/FLI-1 fusion t-transcript detection and MIC2 immunohistochemical staining in the diagnosis of Ewing's sarcoma. Pediatr Pathol Lab Med 1996;16:379-92.

8. Alvarez-Berdecia A, Schut L, Bruce DA. Localised primary intracranial Ewing's sarcoma of the orbital roof. J Neurosurg 1979;50:811-3.

9. Woodruff G, Thorner P, Skarf B. Primary Ewing's sarcoma of the orbit presenting with visual loss. Br J Ophthalmol 1988;72:786-92.

10. Yamada S, Takahashi H. A case of Ewing's tumour (reticulum cell sarcoma) of the orbit. Hirosake Med J 1957;8:202-7.

11. Howard DJ, Lund VJ. Primary Ewing's sarcoma of the ethmoid bone. J Laryngol Otol 1985;99:1019-23.

12. McManus AP, Gusterson BA, Pinkerton CR, Shipley JM. The molecular pathology of small round-cell tumours: relevance to diagnosis, prognosis, and classification. J Pathol 1996;178:116-21.

13. Schmidt D, Herrmann C, Jurgens H, Harms D. Malignant peripheral neuroectodermal tumour and its necessary distinction from Ewing's sarcoma: a report from the Kiel Pediatric Tumour Registry. Cancer 1991;68:2251-9.

14. Siebenrock KA, Nascimento AG, Rock MG. Comparison of soft tissue Ewing's sarcoma and peripheral neuroectodermal tumour. Clin Orthop 1996;329:288-99.

15. Wilkins RM, Pritchard DJ, Burgert EO Jr, Unni KK. Ewing's sarcoma of bone. Experience with 140 patients. Cancer 1986;58:2551-5.

16. Dunst J, Sauer R, Burgers JMV, et al. Radiation therapy as local treatment in Ewing's sarcoma: results of the cooperative Ewing's Sarcoma Studies CESS 81 and CESS 86. Cancer 1991;67:2818-25.

17. Burgert EO Jr, Nesbit ME, Garnsey LA, et al. Multimodal therapy for the management of nonpelvic, localised Ewing's sarcoma of bone: intergroup study IESS-II. J Clin Oncol 1990;8:1514-24.

18. Tewari MK, Sharma BS, Banerjee AK, et al. Primary Ewing's sarcoma of the orbit. Ind Pediatr 1993;30:930-3.

19. Fiorillo A, Tranfa F, Canale G, et al. Primary Ewing's sarcoma of the maxilla, a rare and curable localisation: report of two new cases, successfully treated by radiotherapy and systemic chemotherapy. Cancer Lett 1996;103:177-82.

20. Rudoler SB, Corn BW, Shields CL, et al. External beam irradiation for choroid metastases: identification of factors predisposing to long-term sequelae. Int J Radiat Oncol Biol Phys 1997;38:251-6.

21. Fontanesi J, Parham DM, Pratt C, Meyer D. Second malignant neoplasm in children with retinoblastoma: the St Jude Children's Research Hospital experience. Ophthalmic Genet 1995;16:105-8. 